\title{
Hipovitaminose A no Brasil: um problema de saúde pública
}

\author{
Rejane Andréa Ramalho, ${ }^{1}$ Hernando Flores $^{2}$ e Cláudia Saunders ${ }^{1}$
}

RESUMO A deficiência de vitamina A é considerada um dos problemas de saúde pública de fácil prevenção mais importantes em diversos países, inclusive o Brasil. Assim, o objetivo do presente trabalho foi revisar a literatura sobre carência de vitamina A publicada entre 1970 e 2000, disponível nas bases de dados MEDLINE e LILACS, e avaliar a hipovitaminose A na América Latina e no Brasil. A pesquisa revelou que até os anos 1980, a atenção dada pela saúde pública à vitamina A se concentrou na importância dessa vitamina para a visão. Na segunda metade dessa década, estudos epidemiológicos sugeriram que, em nível populacional, a deficiência subclínica de vitamina A também poderia ser deletéria para certas etapas do metabolismo, com grande influência sobre os indices de morbidade e mortalidade infantil. Em todas as regiões brasileiras para as quais existem dados, foi constatada a carência marginal de vitamina A, com alta prevalência em diferentes faixas etárias, o que não se justifica com a tecnologia e os recursos atualmente disponíveis. É preciso que se assuma o compromisso de reduzir a deficiência de vitamina A para garantir o desenvolvimento adequado das próximas gerações.

Palavras-chave Deficiência de vitamina A, indicadores, programas e políticas de nutrição e alimentação, inquéritos nutricionais.

Até a segunda metade da década de 1980, a deficiência de vitamina A (DVA) causava preocupação apenas em relação a seus sinais clínicos, que vão desde a cegueira noturna até a cegueira nutricional irreversível. $\mathrm{Na}$ segunda metade dessa década surgiram evidências de que a carência sub-clínica da vitamina A, sem sinais como xeroftalmia, mancha de Bitot e ceratomalacia,

\footnotetext{
Universidade Federal do Rio de Janeiro (UFRJ), Instituto de Nutrição Josué de Castro, Departamento de Nutrição e Dietética. Correspondência e pedidos de separatas devem ser enviados a Rejane Andréa Ramalho no seguinte endereço: Centro de Ciências da Saúde, Bloco J, $2^{\circ}$ andar, Universidade Federal do Rio de Janeiro, Ilha do Fundão, CEP 21944-970, Rio de Janeiro, RJ, Brasil. Telefone/fax: +55-21-2280-8343; e-mail: aramalho@rionet.com.br

2 Universidade Federal de Pernambuco, Departamento de Nutrição, Recife, PE, Brasil.
}

também pode contribuir para a morbidade e mortalidade em crianças, recémnascidos e mulheres em idade fértil, puérperas e nutrizes, os grupos tradicionalmente considerados de risco. Atualmente, sabe-se que, em função de sua atuação no olho e no ciclo visual, a DVA pode tornar mortais doenças como o sarampo, considerada parte do histórico de saúde de crianças normais. De fato, a DVA pode provocar quadros de imunodeficiência de origem exclusivamente nutricional (1).

Existem evidências de que os programas de intervenção nutricional, ainda que não integrados a outros programas de intervenção em saúde e nutrição, podem evitar, cada ano, a morte de até 2,5 milhões de crianças; salvar da cegueira nutricional irreversível aproxi- madamente 500000 crianças; e livrar dessa síndrome de deficiência imunológica nutricional quase 1 bilhão de pessoas (prevalência anual estimada). Tais evidências motivaram conferências como a Cumbre Mundial sobre la Infancia, de 1990, onde os governos firmaram um compromisso político de dar atenção prioritária às deficiências nutricionais, entre elas as de micronutrientes; a Reunião Internacional de Montreal, de 1991; e a Conferência Internacional de Nutrición, de 1992 (1). Essas três reuniões geraram dois documentos principais: a Declaração Mundial sobre Nutrição e o Plano de Ação para Melhorar a Nutrição, ratificados pelo governo brasileiro e por mais de uma centena de países nos quais a DVA é endêmica (2). 
Por seu impacto na produtividade da população e por elevar significativamente os gastos da rede de saúde (3), a DVA representa um entrave para o desenvolvimento dos países do Terceiro Mundo. A Organização Pan-Americana da Saúde (OPAS) reconhecia, em 1999, a escassa probabilidade de eliminar a cegueira nutricional e outras conseqüências da carência de vitamina A até o ano de 2000 (4). Declarava ainda que a xeroftalmia clínica havia diminuído para cerca de 3 milhões de casos por ano no mundo. No entanto, a prevalência da deficiência sub-clínica teria aumentado. É importante lembrar que tais números representam apenas estimativas, e não o resultado de inquéritos de carência clínica ou sub-clínica, e que, portanto, o "aumento" da carência sub-clínica poderia ser efeito da maior informação disponível sobre a carência marginal.

Após analisar dados do período de 1990 a 1995, as autoridades de saúde no Brasil consideraram que a informação é insuficiente para definir a situação da carência de vitamina A no país. Atualmente, $23 \%$ das mortes por diarréia em crianças brasileiras são reconhecidamente associadas à DVA (5). Medidas regionais de intervenção vêm sendo implementadas através da distribuição de megadoses de vitamina A durante campanhas de multivacinação e através de ações educativas em áreas endêmicas como parte do Programa Nacional da Deficiência de Vitamina A (6).

O presente trabalho relata os resultados de uma revisão da literatura sobre a carência de vitamina A publicada de 1970 a 2000, disponível nas bases de dados MEDLINE e LILACS, com ênfase na América Latina e, em especial, no Brasil. O foco desta revisão foi avaliar a hipovitaminose A em seu contexto epidemiológico, além de abordar a necessidade do emprego efetivo de todos os recursos humanos, científicos e tecnológicos disponíveis para o combate dessa carência nutricional como estratégia vital para a sobrevivência das crianças e para o desenvolvimento dos países afetados.

\section{EPIDEMIOLOGIA DA DEFICIÊNCIA DE VITAMINA A}

A DVA constitui um problema grave em mais de 60 países (7). Sua prevalência é particularmente alta em regiões como a Ásia, África e América Latina, ainda que os inquéritos nacionais sejam escassos. O Brasil foi classificado pela Organização Mundial da Saúde (OMS) e pela OPAS $(3,4)$ como área de carência sub-clínica grave. Stephens et al. (8) chamam a atenção para a subestimação do número de casos de carência sub-clínica de vitamina A inclusive em países desenvolvidos, como os Estados Unidos, onde a ingestão inadequada de retinol e carotenóides é o principal fator etiológico da DVA.

Considera-se atualmente que os casos de xeroftalmia representam apenas a ponta do iceberg, sob a qual podem se encontrar diferentes proporções (naturalmente muito maiores) da população em estágios menos avançados (marginais) de carência (9). Durante muito tempo, o papel da carência de vitamina A em outras funções metabólicas - na reprodução, no sistema imunológico e na manutenção da integridade epitelial - foi quase ignorado, possivelmente devido à falta de indicadores sub-clínicos ou pré-patológicos da hoje chamada carência marginal (10-12). Calcula-se que o número de crianças com carência marginal de vitamina A seja de cinco a 10 vezes maior do que o número de crianças com carência clínica $(13,14)$.

As observações epidemiológicas que relacionam a deficiência marginal de vitamina $\mathrm{A}$ ao aumento das taxas de morbidade e mortalidade infantil têm levado ao desenvolvimento de métodos diagnósticos cada vez mais sensíveis e precoces, como a resposta relativa à dose (relative dose response, RDR); o teste modificado de resposta relativa à dose (modified relative dose response, MRDR); a concentração de vitamina A no leite humano; a avaliação histológica através da citologia de impressão conjuntival; e a avaliação fisiológica através do diagnóstico da cegueira noturna $(4,15)$. Atualmente, é imprescin- dível a utilização desses indicadores. É preciso ter cuidado com a noção de que existem regiões onde a xeroftalmia é rara, já que são escassos os dados de prevalência das formas clínicas. De fato, praticamente nenhum país dispõe de um inquérito nacional sobre DVA que permita, a rigor, descartar esse problema como sendo da ordem da saúde pública.

\section{Deficiência de vitamina A na América Latina e no Caribe}

A OMS (16) e a OPAS (4) definiram, para os diversos indicadores de deficiência de vitamina $\mathrm{A}$, os limites de prevalência acima dos quais o problema adquire magnitude de problema de saúde pública. Para sinais clínicos, as prevalências aceitáveis são: $1,0 \%$ para cegueira noturna $(\mathrm{XN}) ; 0,5 \%$ para mancha de Bitot $(X 1) ; 0,01 \%$ para xerose da córnea ou ulceração da córnea $(\mathrm{X} 2, \mathrm{X} 3 \mathrm{~A}, \mathrm{X3B})$; e 0,001\% para cicatriz da córnea $(\mathrm{XS})$.

Essa classificação tem maior valor teórico do que prático, visto que poucos países têm condições técnicas e financeiras de realizar inquéritos clínicos - já que, para descartar a hipótese de o problema ter "magnitude de saúde pública", tais estudos necessitariam de amostras aleatórias que podem chegar a 4,4 milhões de crianças (tabela 1) (17). Com tais indicadores, muitas áreas nunca submetidas a inquéritos clínicos não são consideradas áreas de risco e não são alvo de ações específicas de intervenção, com exceção do Programa de Fortificação de Açúcar na América Central, principalmente na Guatemala, $\mathrm{Pa}$ namá, Nicarágua e Honduras, do Instituto de Nutrición de Centroamérica y Panamá (INCAP), e de alguns projetos de pequeno porte na Bolívia e na região Nordeste do Brasil $(9,18)$.

Os únicos inquéritos clínicos de alguma significância em termos de tamanho amostral foram realizados na Ásia (19-21), o que explica, mas não justifica, a freqüente afirmação de que o problema só tem importância clínica naquele continente. De fato, Ásia e 
TABELA 1. Marcadores de carência de vitamina A: amostra populacional necessária para realizar inquéritos com o objetivo de avaliar o problema como sendo de saúde pública ${ }^{\mathrm{a}}$

\begin{tabular}{lcr}
\hline \multicolumn{1}{c}{ Indicadores } & $\begin{array}{c}\text { Prevalência máxima } \\
\text { aceita (\%) }\end{array}$ & $\begin{array}{c}\text { Número de } \\
\text { indivíduos na } \\
\text { amostra }\end{array}$ \\
\hline Xerose da córnea & 0,001 & 4444044 \\
Cicatriz da córnea & 0,05 & 807681 \\
Mancha de Bitot & 0,5 & 87628 \\
Cegueira noturna & 1,0 & 4604 \\
Retinol sérico $<0,35 \mu \mathrm{mol} / \mathrm{L}$ & 5,0 & 8411 \\
Retinol no leite materno $<0,70 \mu \mathrm{mol} / \mathrm{L}$ & 15,0 & 2535 \\
RDR, MRDR, S30DR, retinol sérico $<1,05 \mu \mathrm{mol} / \mathrm{L}^{\mathrm{b}}$ & 20,0 & 1800 \\
\hline
\end{tabular}

a Indicadores dispostos por ordem de prevalência para que o problema tenha magnitude de saúde pública. 0 tamanho da amostra é o necessário para que o limite superior de confiança de 95\% fique abaixo do máximo estipulado, assumindo um erro de estimativa de $15 \%$.

${ }^{\mathrm{b}} \mathrm{RDR}=$ resposta relativa à dose; MRDR = teste modificado de resposta à dose relativa; $\mathrm{S} 30 \mathrm{DR}$ = resposta sérica de 30 dias.
África são as regiões onde se concentram as maiores prevalências mundiais conhecidas de xeroftalmia, por ser as únicas nas quais existem estimativas de prevalência de sinais clínicos. Apesar disso, há pelo menos 10 países na América Latina (incluindo o Brasil) e no Caribe que são considerados áreas de risco $(19,20,22-24)$ e que estão incluídos no mapa da DVA traçado pela OMS (tabela 2).

\section{Deficiência de vitamina A no Brasil}

A informação sobre DVA em Brasil provém de inquéritos nutricionais em diversas regiões e grupos populacionais (36). Os dados dos últimos 20 anos indicam que a DVA é um problema com magnitude de saúde pública em todo o país.

Os inquéritos de consumo alimentar realizados nos últimos 25 anos em âm-
TABELA 2. Prevalência de níveis inadequados de retinol sérico em países da América Latina e do Caribe

\begin{tabular}{lccc}
\hline \multicolumn{1}{c}{ País } & Faixa etária & Prevalência (\%) & Ano e fonte \\
\hline Argentina & & & \\
$\quad$ Buenos Aires & $<3$ anos & $25^{\mathrm{a}}$ & $1998(25)$ \\
Províncias & $<3$ anos & $35^{\mathrm{a}}$ & $1998(25)$ \\
Bolívia, La Paz & 1 a 5 anos & $11^{\mathrm{a}}$ & $1991(26)$ \\
Brasil, Nordeste & 2 a 8 anos & $49^{\mathrm{b}}$ & $1992(9)$ \\
El Salvador & 0 a 5 anos & $36^{\mathrm{b}}$ & $1993(27)$ \\
Equador & 0 a 4,9 anos & $14^{\mathrm{b}}$ & $1992(9)$ \\
$\quad$ Urbano & 0 a 5 anos & $16^{\mathrm{b}}$ & $1992(9)$ \\
Rural & 0 a 5 anos & $12^{\mathrm{b}}$ & $1992(9)$ \\
Guatemala & 1 a 7 anos & $21^{\mathrm{b}}$ & $1993(28)$ \\
Honduras & Pré-escolares & $40^{\mathrm{a}}$ & $1999(29)$ \\
& Escolares & $34^{\mathrm{a}}$ & $1999(29)$ \\
México & Pré-escolares & $32^{\mathrm{b}}$ & $1994(30)$ \\
Nicarágua & $<4$ anos & $30^{\mathrm{b}}$ & $1993(31)$ \\
Panamá & 1 a 4,9 anos & $28^{\mathrm{b}}$ & $1993(32)$ \\
Peru & & & $1993(33)$ \\
$\quad$ Urbano & $<6$ anos & $15^{\mathrm{b}}$ & $1993(33)$ \\
$\quad$ Rural & $<6$ anos & $33^{\mathrm{b}}$ & $1993(34)$ \\
República Dominicana, Sudoeste & 1 a 5 anos & $20^{\mathrm{a}}$ & $1998(35)$ \\
Venezuela & $<6$ anos & $33^{\mathrm{b}}$ & \\
\hline
\end{tabular}

\footnotetext{
a Retinol sérico $<0,70 \mu \mathrm{mol} / \mathrm{L}$.
}

${ }^{b}$ Retinol sérico $<1,05 \mu \mathrm{mol} / \mathrm{L}$. bito nacional, regional ou local indicam que a ingestão de vitamina $\mathrm{A}$ de fontes naturais (incluindo provitamina A) é extremamente baixa em $60 \%$ ou mais da população, com uma adequação inferior a 50\% (36-41). A OPAS (4) reconhece que, da ingestão média total de vitamina A na América do Sul, $50 \%$ provêm de fontes vegetais (provitamina A, carotenóides). É importante ressaltar que nesses $50 \%$ estão incluídos os carotenóides sem atividade de provitamina A, como o licopeno, a luteína e a zeaxantina (42). A monotonia alimentar prevalente entre as diversas camadas da população, principalmente as mais pobres $(43,44)$, contribui para a manutenção do quadro que prevalece até hoje.

Em termos epidemiológicos, a ingestão inadequada de alimentos que são fonte de vitamina A é o principal fator etiológico em todas as áreas endêmicas no mundo (principalmente de populações carentes de países em desenvolvimento na América Latina, África e Ásia, nas quais está incluído o Brasil) $(2,3,18)$. Tal fato foi constatado há mais de 30 anos por Reh (45), e confirmado por vários estudos posteriores $(27,31,36,37,39-41,46)$. Além disso, nas mesmas áreas há fatores coadjuvantes, como a reduzida ingestão de lipídios (que aumentam a biodisponibilidade da vitamina e provitamina A), alta prevalência de infecções, falta de saneamento ambiental e de água tratada, condições socioeconômicas desfavoráveis e tabus alimentares que aumentam a demanda ou interferem na ingestão e metabolização da vitamina $\mathrm{A}$ pelo organismo $(3,4,47,48)$. A biodisponibilidade dos carotenóides também depende de outros nutrientes cuja ingestão é geralmente deficiente nas populações afetadas, tais como proteínas, zinco, vitamina E e fibras $(4,42)$.

No Brasil, os inquéritos bioquímicos disponíveis confirmam que a DVA é um problema de saúde pública nos estados de São Paulo, Minas Gerais, Pernambuco, Paraíba, Ceará, Bahia e Amazonas (tabela 3). No Rio de Janeiro também foram observadas altas prevalências de DVA. Em gestantes consideradas de baixo risco, a inadequação dietética atinge $12 \%$ (71). Accioly et al. 
(60) observaram inadequação dos níveis séricos de retinol em 13\% das gestantes no $3^{\circ}$ trimestre de gestação e $15 \%$ de inadequação na ingestão dietética de vitamina A nesse mesmo período gestacional, tendo sido observada associação significativa entre o indicador dietético e o bioquímico. Ramalho et al. (58) encontraram inadequação dos níveis séricos de retinol em puérperas (24\%) e em seus recém-nascidos (56\%). Observou-se ainda que a hipovitaminose A pode ocorrer em mães e recém-nascidos independentemente do estado antropométrico materno pré-gestacional e do ganho de peso durante a gestação (72).

Apesar de os inquéritos hoje disponíveis no Brasil não terem caráter nacional, eles são unânimes em apontar um problema grave de carência marginal de vitamina A (tabela 3).

\section{CONCLUSÕES}

A hipovitaminose A tem conseqüências não apenas para a visão, mas também para diversas funções orgânicas. Sabe-se que os indicadores clínicos não permitem o correto dimensionamento do problema para fins preventivos. Através do estudo de populações em que a deficiência é endêmica, assim como do acompanhamento de programas de intervenção, sabe-se também que essa carência responde por uma parcela importante dos índices de morbidade e mortalidade infantil.

Embora a deficiência de vitamina A se concentre mais no Terceiro Mundo, sabe-se que ela não é exclusiva de áreas geográficas economicamente desfavorecidas. No Brasil, pensava-se que o problema estaria limitado às regiões mais pobres do Norte e Nordeste, mas os dados da região Sudeste em nada diferem dos dados dessas regiões, tornando a DVA independente do mapa econômico do país.

Com o desenvolvimento de marcadores de carência marginal mais precoces e de menor custo não parece haver justificativa para que qualquer país ou região ainda apresente alta prevalência de DVA por falta de programas de intervenção nutricional de abrangência nacional. Isso inclui as várias regiões

TABELA 3. Prevalência de níveis inadequados de retinol sérico em diversas regiões do Brasil

\begin{tabular}{|c|c|c|}
\hline Localidade & Prevalência (\%) & Ano e fonte \\
\hline \multicolumn{3}{|l|}{ Cidade de São Paulo } \\
\hline 2 a 7 anos & $51^{a}$ & $1978(49)$ \\
\hline 15 a 60 anos & 19 a $32^{a}$ & $1975(50)$ \\
\hline 1 a 13 anos & $59^{b}$ & $1980(51)$ \\
\hline 2 a 6 anos & $74^{\mathrm{a}}$ & $1980(51)$ \\
\hline \multicolumn{3}{|c|}{ Outras regiões do Estado de São Paulo } \\
\hline 2 a 6 anos & $30^{\mathrm{a}}$ & $1984(52)$ \\
\hline 2 a 6 anos & $49^{a}$ & $1981(53)$ \\
\hline 7 a 10 anos & $35^{\mathrm{a}}$ & $1981(53)$ \\
\hline 2 a 8 anos & 49 a $95^{b}$ & $1986(54)$ \\
\hline Todas as idades & $18^{\mathrm{a}}$ & $1972(36)$ \\
\hline$>50$ anos & $6^{\mathrm{a}}$ & $1985(55)$ \\
\hline 3 a 10 anos & $18^{\mathrm{a}}$ & $1995(56)$ \\
\hline \multicolumn{3}{|l|}{ Cidade do Rio de Janeiro } \\
\hline Recém-nascidos & $56^{\mathrm{b}}$ & $1999(57)$ \\
\hline Puérperas & $23^{b}$ & $1998(58)$ \\
\hline$<6$ anos & $35^{\mathrm{b}}$ & $2001(59)$ \\
\hline Gestantes & $13^{\mathrm{b}}$ & $2001(60)$ \\
\hline \multicolumn{3}{|l|}{ Cidade de Belo Horizonte } \\
\hline Pré-escolares & $22^{\mathrm{a}}$ & $1987(61)$ \\
\hline 0 a 7 anos & $94^{\mathrm{a}}$ & $1994(62)$ \\
\hline \multicolumn{3}{|c|}{ Outras regiões do Estado de Minas Gerais } \\
\hline Pré-escolares & $34^{\mathrm{a}}$ & $1986(63)$ \\
\hline Adultos & $60^{\mathrm{a}}$ & $1988(64)$ \\
\hline \multicolumn{3}{|l|}{ Cidade do Recife } \\
\hline 2 a 6 anos & $34^{\mathrm{a}}$ & $1991(43)$ \\
\hline 2 a 5 anos & $40^{\mathrm{b}}$ & $1982(65)$ \\
\hline 18 a 85 anos & $12^{\mathrm{a}}$ & $1984(66)$ \\
\hline \multicolumn{3}{|c|}{ Outras regiões do Estado de Pernambuco } \\
\hline 1 a 7 anos & $40^{\mathrm{b}}$ & $1993(67)$ \\
\hline 0 a 5 anos & $40^{\mathrm{a}}$ & $1993(68)$ \\
\hline Gestantes & $3^{a}$ & $1971(39)$ \\
\hline \multicolumn{3}{|l|}{ Cidade de João Pessoa } \\
\hline 1,2 a 6 anos & $18^{\mathrm{a}}$ & $1991(43)$ \\
\hline \multicolumn{3}{|c|}{ Estado da Paraíba, semi-árido } \\
\hline 1 a 4,9 anos & $16^{\mathrm{a}}$ & $1991(43)$ \\
\hline Pré-escolares & $21^{a}$ & $1996(69)$ \\
\hline \multicolumn{3}{|l|}{ Cidade de Fortaleza } \\
\hline 0,5 a 4,9 anos & $40^{\mathrm{a}}$ & $1991(43)$ \\
\hline \multicolumn{3}{|c|}{ Outras regiões do Estado do Ceará } \\
\hline 0,5 a 4,9 anos & $31^{a}$ & $1991(43)$ \\
\hline \multicolumn{3}{|l|}{ Estado da Bahia } \\
\hline 0 a 6 anos & $55^{\mathrm{a}}$ & $1991(43)$ \\
\hline \multicolumn{3}{|l|}{ Estado da Bahia, semi-árido } \\
\hline Pré-escolares & $55^{\mathrm{a}}$ & $1996(69)$ \\
\hline \multicolumn{3}{|l|}{ Cidade de Manaus } \\
\hline Pré-escolares & $36^{\mathrm{a}}$ & $1988(70)$ \\
\hline
\end{tabular}

${ }^{a}$ Retinol sérico $<0,70 \mu \mathrm{mol} / \mathrm{L}$.

${ }^{b}$ Retinol sérico $<1,05 \mu \mathrm{mol} / \mathrm{L}$.

da América Latina e, especialmente, o Brasil. A erradicação ou, pelo menos, a redução das taxas de hipovitaminose $\mathrm{A}$ teria um impacto social positivo e deve constituir um compromisso ético com as próximas gerações. O Banco Mundial calcula que o custo de não intervir para superar a desnutrição e, principalmente, as carências específicas, é 10 vezes maior do que o custo de progra- mas de intervenção (73). Mesmo assim, não é raro que os setores envolvidos na busca de uma solução - a universidade, os governos, a indústria, a mídia, e a população - falem linguagens diferentes. Precisamos, então, de interfaces entre esses setores para permitir que o conhecimento científico se traduza em ações e programas de intervenção nutricional de alcance social. 


\section{REFERÊNCIAS}

1. Vitamin A Field Support Project (VITAL). Tercer taller regional sobre deficiencias de vitamina A y otros micronutrientes en América Latina y el Caribe (Recife, Brasil). Arlington, Virgínia: VITAL/US Agency for International Development; 1993.

2. Organización Mundial de la Salud (OMS). Prevención y tratamiento de la carencia de vitamina A y de la xeroftalmia. Ginebra: OMS; 1982. (Série Informes Técnicos, 672).

3. World Health Organization (WHO). Global prevalence of vitamin A deficiency - micronutrient deficiencies information system. Working paper no 2. Geneva: WHO; 1995. (Document WHO/NUT/95.3).

4. McLaren D, Frigg M. Manual de ver y vivir sobre los trastornos por deficiencia de vitamina A (VADD). Washington: OPAS/OMS; 1999

5. Brasil, Ministério da Saúde. Secretaria de projetos especiais de saúde: metas da cúpula mundial em favor da infância - avaliação de meia década, 1990-1995. Brasília: Ministério da Saúde; 1997.

6. Brasil, Ministério da Saúde, Secretaria de Políticas de Saúde. Política nacional de alimentação e nutrição. Brasília: Ministério da Saúde; 2000.

7. Stoltzfus RJ, Underwood BA. Breastmilk vitamin $\mathrm{A}$ as an indicator to assess vitamin A status of women and infants. Bull World Health Organ 1995;73(5):703-711.

8. Stephens D, Jackson PL, Gutierrez Y. Subclinical vitamin A deficiency. A potentially unrecognized problem in the United States. Pediatr Nurs 1996;22(5):377-456.

9. Mora JO. Deficiencia de vitamina A en América Latina y Caribe: una reevaluación de la situación. Vital News 1992;3(1):1-2.

10. Azaïs-Braesco V, Pascal G. Vitamin A in pregnancy: requirements and safety limits. Am J Clin Nutr 2000;71(5):1325S-1333S.

11. Barreto ML, Santos LP, Assis AO, Araújo MPN, Farenzena GG, Santos PAB, et al. Effect of vitamin A supplementation on diarrhoea and acute lower-respiratory-tract infections in young children in Brazil. Lancet 1994;344 (8917):228-231.

12. Saunders C, Ramalho RA, Leal MC. Estado nutricional de vitamina A no grupo maternoinfantil. Rev Bras Saude Materno-Infantil 2001;1(1):21-29.

13. Fawzi WW, Chalmers TC, Herrera MG, Mosteller F. Vitamin A supplementation and child mortality - a meta-analysis. JAMA 1993;269(7):898-903.

14. Glasziou PP, Mackerras DEM. Vitamin A supplementation in infectious diseases: a metaanalysis. BMJ 1993;306(6874):366-370.

15. World Health Organization (WHO). Indicators for assessing vitamin A deficiency and their application in monitoring and evaluating intervention programmes. Geneva: WHO; 1996.

16. World Health Organization (WHO). Vitamin A supplements: a guide to their use in the treatment and prevention of vitamin A deficiency and xerophthalmia. Geneva: WHO; 1988.
17. Ramalho RA. Deficiência marginal de vitamina A em puérperas, recém-nascidos e préescolares atendidos em três serviços de saúde do Município do Rio de Janeiro [tese de doutorado]. Rio de Janeiro: Escola Nacional de Saúde Pública, Fundação Oswaldo Cruz; 1998.

18. Underwood BA. Estrategias a largo plazo para el control de las deficiencias de micronutrientes. Em: Vitamin A Field Support Project (VITAL). Arlington, Virginia: USAID; 1993. Pp.70-76.

19. Sommer A, Tarwotjo I, Djunaedi E, West KP Loeden A, Tilden R. Impact of supplementation on childhood mortality: a randomized controlled community trial. Lancet 1986; 1(8491):1169-1173.

20. Rahmathullah L, Underwood BA, Thulasiraj RD, Milton RC. Diarrhea, respiratory infections and growth are not affected by a weekly low dose vitamin A supplement: a masked, controlled field trial in children in Southern India. Am J Clin Nutr 1991;54(3):568-577.

21. Reddy V. Control of vitamin A deficiency and blindness. Acta Paediatr Scand Suppl (Sweden) 1991;374:30-37.

22. Milton R, Reddy V, Maidu A. Mild vitamin A deficiency and childhood morbidity: an Indian experience. Am J Clin Nutr 1987;46(5): 827-829.

23. Sommer A, Katz J, Tarwotjo I. Increased risk of respiratory disease and diarrhea in children with preexisting mild vitamin A deficiency. Am J Clin Nutr 1984;40(5):1090-1095.

24. Vijayaraghavan K, Radhaiah G, Surya PB, Rameshwar SKV, Reddy V. Effect of massive dose vitamin A on morbidity and mortality in Indian children. Lancet 1990;336(8727): 1342-1345.

25. Escobal N, Lejarraga $\mathrm{H}$, Acosta L, Rio ME, Portela ML. Vitamin A deficiency in 0.5 to $2.11 \mathrm{yr}$ old children in three urban areas of Argentina. Em: XVII International Vitamin A Consultative Group Meeting. Cairo, Egypt; 1998. P. 43.

26. Vitamin A Field Support Project (VITAL), US Agency for International Development (USAID). Encuesta de vitamina A y encuesta de consumo - áreas deprimidas. Bolívia: VITAL/USAID; 1991.

27. Hernández JF, Orellana H. Diagnóstico y control de las deficiencias de micronutrientes en El Salvador. Em: Vitamin A Field Support Project (VITAL). Arlington, Virginia: USAID; 1993. Pp. 57-58.

28. Bulux J, Hernandez N. Situación de las deficiencias de micronutrientes en Guatemala. Em: Vitamin A Field Support Project (VITAL). Arlington, Virginia: USAID; 1993. Pp. 55-56.

29. Nestel P, Melara A, Rosado J, Mora JO. Vitamin A deficiency and anemia among children 12-71 months old in Honduras. Rev Panam Salud Publica 1999;6(1):34-43.

30. Mora JO, Dary O. Deficiencia de vitamina A y acciones para su prevención y control en América Latina y el Caribe. Bol Oficina Sanit Panam 1994;117(6):519-527.
31. Navas EG, Fletes C. Situación actual de la deficiencia de micronutrientes en Nicaragua Em: Vitamin A Field Support Project (VITAL). Arlington, Virginia: USAID; 1993. Pp. 59-60.

32. Caballero E, Rivera G. Prevalência de deficiência de vitamina A em pré-escolares em Panamá. Em: Vitamin A Field Support Project (VITAL). Arlington, Virginia: USAID; 1993. Pp. 61.

33. Aguila R. Situación de la vitamina A en el Perú. Em: Vitamin A Field Support Project (VITAL). Arlington, Virginia: USAID; 1993. Pp. 53-54.

34. Pan American Health Organization (PAHO), World Health Organization (WHO). Plan of action for the elimination of vitamin A deficiency from the Americas. Washington DC: $\mathrm{PAHO} / \mathrm{WHO} ; 1992$.

35. Solano L, Paez M, Sanchez L, Portillo Z, Ramos G, Callegari C. Vitamin A status of preschool children from a community at nutritional risk. Em: XVII International Vitamin A Consultative Group Meeting. Cairo, Egypt; 1998. P. 43

36. Roncada MJ. Hipovitaminose A: níveis séricos de vitamina A e caroteno em populações litorâneas do Estado de São Paulo, Brasil. Rev Saude Publica 1972;6(1):3-18.

37. Fundação Instituto Brasileiro de Geografia e Estatística (IBGE). Perfil estatístico de crianças e mães no Brasil: aspectos nutricionais, 197475. Rio de Janeiro: IBGE/UNICEF; 1982.

38. Roncada MJ, Mazzilli RN. Fontes de vitamina nas dietas de populações de São Paulo, Brasil. Alimentação Nutrição 1989;1(1):71-86.

39. Batista-Filho M, Chaves N, Varela RM, Souza Martins MH, Salzano AC, Bazante MO, et al. Inquérito nutricional em área urbana da Zona das Mata do Nordeste Brasileiro - Água Preta — Pernambuco. Hospital 1971;79(5): 139-155.

40. Deus MB, Coelho HAL, Romani SAM. Consumo alimentar de Pernambuco. Recife: Universidade Federal de Pernambuco; 1979.

41. Trigo M. Análise de situação alimentar de dois núcleos populacionais de Marabá, Pará. Alimentação 1985;80(5):17-27.43.

42. Saunders C, Ramalho A, Accioly E, Paiva F. Utilização de tabelas de composição de alimentos na avaliação do risco de hipovitaminose A. Arch Latinoam Nutr 2000;50(3): 237-242.

43. McAuliffe J, Santos LM, Diniz AS, BatistaFilho M, Barbosa RCC. A deficiência de vitamina A e estratégias para o seu controle: um guia para as Secretarias Municipais de Saúde. Fortaleza: Project HOPE; 1991

44. Diniz AS, Hilderbrand K. A study on the effectiveness of adding zinc to vitamin A supplements in the prevention of vitamin A deficiency. Sight and Life Newsletter 1997;2:15.

45. Reh J. La ingestión de vitamina A en Latinoamérica y el Caribe. Bol Oficina Sanit Panam 1970;68(1):53-63.

46. International Vitamin A Consultative Group (IVACG). Guidelines for the development of a simplified assessment to identify groups at 
risk for inadequate intake of vitamin A. New York: The Nutrition Foundation; 1989.

47. Dimitrov NV, Meyer C, Ullrey DE. Bioavailability of $\beta$-carotene in humans. Am J Clin Nutr 1988;48(2):298-304.

48. Ramalho RA, Saunders C. O papel da educação nutricional no combate às carências nutricionais. Rev Nutrição 2000;13(1):11-16.

49. Roncada MJ, Wilson D, Netto AL, Netto OB, Kalil AC, Nunes MF, et al. Hipovitaminose A em filhos de migrantes nacionais em trânsito pela capital do Estado de São Paulo, Brasil: estudo clínico-bioquímico. Rev Saude Publica 1978;12(3):345-350.51.

50. Roncada MJ. Inquérito entre migrantes atendidos pela central de triagem e encaminhamento, na capital do Estado de São Paulo, Brasil II. Aspectos bioquímicos da hipovitaminose A. Rev Saude Publica 1975;9(3):313329.

51. Wilson D, Roncada MJ, Lui Netto A, Berretta N O. Hipovitaminose A em pré-escolares internados em uma instituição na capital do Estado de São Paulo, Brasil. Rev Saude Publica 1981;15(4):395-400.

52. Roncada MJ, Wilson D, Okani ET, Aminos S. Prevalência da hipovitaminose A em préescolares do município de área metropolitana de São Paulo. Rev Saude Publica 1984;18(3): 218-224.

53. Roncada MJ, Wilson D, Mazzilli RN, Gandra YR. Hipovitaminose A em comunidades do Estado de São Paulo, Brasil. Rev Saude Publica 1981;15(3):338-349.

54. Favaro RMD, Souza NV, Batista SM, Ferriani MGC, Desai ID, Dutra de Oliveira JE. Vitamin A status of young children in southern Brazil. Am J Clin Nutr 1986:43(5):852-858.

55. Roncada MJ, Marucci MF, Lamonica JM. Serum levels of Vitamin A and carotene in middle-aged and aged subjects, in 11 localities of the State of São Paulo, Brazil. Rev Saude Publica 1985;19(4):336-343.
56. Carvalho CMG, Farfan JA, Wilke BC, Vencovsky R. Prevalência de hipovitaminose A em crianças da periferia do Município de São Paulo, Brazil. Cad Saude Publica 1995;11(1): 85-96.

57. Ramalho RA, Anjos LA, Flores H. Níveis de Retinol no sangue materno e no cordão umbilical de seus recém-nascidos no Rio de Janeiro. Arch Latinoam Nutr 1999;49(4):318- 321.

58. Ramalho RA, Anjos LA, Flores H. Hipovitaminose $\mathrm{A}$ em recém-nascidos em duas maternidades públicas no Rio de Janeiro. Cad Saude Publica 1998;14(4)821-827.

59. Ramalho RA, Anjos LA, Flores H. Estado nutricional de vitamina A e teste terapêutico em pré-escolares no Rio de Janeiro. Rev Nutrição 2001;14(1)23-28.

60. Accioly E, Souza-Queiróz S. Deficiencia de vitamina A en embarazadas asistidas en una maternidad pública en Rio de Janeiro, Brasil. Rev Chilena Nutrición 2001;27(3):352-357.

61. Araújo RL, Araújo MBDG, Machado RDP, Braga AA, Leite BV, Oliveira JR. Evaluation of a program to overcome vitamin $\mathrm{A}$ and iron deficiencies in areas of poverty in Minas Gerais, Brazil. Arch Latinoam Nutr 1987;37(1): 9-22.

62. Dutra de Oliveira JE, Marchini JS. Levantamento bibliográfico de estudos bioquímicosnutricionais sobre micronutrientes realizados no Brasil. Cadernos Nutrição 1994;8(1):31-67.

63. Araújo RL, Araújo MBDG, Sieiro RO, Machado RDP, Leite BV. Diagnóstico da situação de hipovitaminose A e da anemia nutricional na população do Vale do Jequetinhonha, Minas Gerais, Brasil. Arch Latinoam Nutr 1986;36(4):642-652.

64. Souza MSL. Inter-relação entre parasitoses e hipovitaminose $\mathrm{A}$ - infecção e o nível sérico de retinol de população de zona endêmica de Minas Gerais/Brasil. Rev Inst Med Trop Sao Paulo 1988;30(4):281-287.
65. Flores $\mathrm{H}$. The relative dose response (RDR) in malnourished children. Xerophthalmia Club Bull 1992;49:4-5.

66. Flores $\mathrm{H}$, Araújo CR. Liver level of retinol in unselected necropsy specimens: a prevalence survey of vitamin A deficiency in Recife, Brazil. Am J Clin Nutr 1984;40(1):146-152.

67. Brunken GS, Flores H. Consumption of vitamin A rich foods. Xerophthalmia Club Bulletin 1993;54:3-4.

68. Flores H. Frequency distributions of serum vitamin A levels in cross-sectional surveys and in surveys before and after vitamin A supplementation. Em: International Vitamin A Consultative Group (IVACG). A brief guide to current methods of assessing vitamin A status. Washington, DC: The Nutrition Foundation Inc; 1993. Pp. 9-11.

69. Santos LMP, Batista-Filho M, Diniz AS. Epidemiologia da carência de vitamina A no Nordeste do Brasil. Bol Oficina Sanit Panam 1996;120(6):525-535.

70. Silva NB. Prevalência de Anemia ferropriva em crianças pré-escolares da cidade de Manaus, AM. Em: Anais do I Congresso Nacional da SBAN. São Paulo, Brasil; 1988. Pp. 10-11.

71. Coelho CSP, Ramalho RA, Accioly E. O inquérito dietético na avaliação do estado nutricional de vitamina A em gestantes. ARS CVRANDI Clínica Médica 1995;6(28):44-60.

72. Ramalho RA, Saunders C, Paiva F, Accioly E, Cardoso LO, Natalizi D. Estado de vitamina A de puérperas e recém-nascidos e estado antropométrico materno. Rev Ciências Médicas 2001;10(1):5-10.

73. World Bank. Enriching lives: overcoming vitamin and mineral malnutrition in developing countries. Washington, DC: The International Bank for Reconstruction and Development; 1994.

Manuscrito recebido em 23 de agosto de 2001. Aceito em

ABSTRACT Vitamin A deficiency is considered one of the most important of the easily preventable public health problems in a number of countries, including Brazil. The objective of this study was to review the scientific literature in the MEDLINE and LILACS databases that was published between 1970 and 2000 concerning vitamin A deficiency, and to assess the occurrence of hypovitaminosis A in Latin America, especially Brazil. Our research showed that until around 1980 the public health concerns focused mainly on the importance of vitamin A in ensuring good vision. In the second half of the 1980s, epidemiological studies suggested that, on a population level, subclinical vitamin A deficiency could also have a negative effect on metabolic functions, with a great impact on childhood morbidity and mortality. Marginal vitamin A deficiency has been reported in all the regions of Brazil for which there are data available, with high prevalences in various age groups. This situation is inexcusable, given the health care technology and resources that are now available. There must be a commitment to reducing vitamin A deficiency in order to ensure the adequate development of future generations. 
versão revisada em 24 de abril de 2002. 